\title{
Characteristics of colorectal cancer that produce positive immunochemical occult blood test results on stool obtained by digital rectal examination
}

\author{
Hidenori Nakama $\mathrm{MD}^{1}$, Bing Zhang MD ${ }^{1}$, ASM Abdul Fattah MD ${ }^{1,2}$, \\ Noboru Kamijo MD ${ }^{3}$, Xing Zhang $\mathrm{MD}^{1}$
}

H Nakama, B Zhang, ASMA Fattah, N Kamijo, X Zhang. Characteristics of colorectal cancer that produce positive immunochemical occult blood test results on stool obtained by digital rectal examination. Can J Gastroenterol 2001;15(4): $227-230$

OBJECTIVE: To clarify the clinicopathological features of colorectal cancer that show positive results on an immunochemical fecal occult blood test of stool obtained by digital rectal examination.

METHODS: In a cross-sectional study, 9952 subjects received both an immunochemical fecal occult blood test of stool obtained by digital rectal examination and colonoscopy annually over a nine-year period of medical checkups; 64 patients with colorectal cancer were identified. The study subjects comprised 39 patients with colorectal cancer who had positive results (positive group) and 25 patients with colorectal cancer who had negative results (negative group) on an immunochemical fecal occult blood test of stool obtained by digital rectal examination. The positive and negative groups were compared in terms of their individual factors, such as site, size, Dukes classification and histological type of the cancer lesions.

RESULTS: The prevalence of rectal cancers was higher in the positive group than in the negative group $(\mathrm{P}<0.05)$, but there were no differences between the two groups with respect to any other factors
CONCLUSIONS: These findings indicate that stool obtained during the digital rectal examination is unsuitable for detecting fecal occult blood, especially for the detection of proximal colon neoplasms.

Key Words: Colorectal cancer; Digital rectal examination; Immunochemical fecal occult blood test

Caractéristiques du cancer colorectal donnant des résultats positifs à la recherche de sang occulte dans les selles obtenus par toucher rectal

OBJECTIF : Clarifier les caractéristiques clinicopathologiques du cancer colorectal après obtention de résultats positifs au dépistage immunochimique de sang occulte dans les selles sur un échantillon recueilli lors du toucher rectal.

MÉTHODES : Dans une étude transversale, 9952 sujets ont subi annuellement pendant une période de neuf ans un test de dépistage immunochimique de sang occulte dans les selles sur un échantillon recueilli lors du toucher rectal et une coloscopie; 64 patients se sont révélés atteints d'un cancer colorectal. Parmi eux, 39 sujets avaient obtenu des résultats positifs et 25 des résultats négatifs au test de

voir page suivante

${ }^{1}$ Department of Public Health, Shinshu University School of Medicine; ${ }^{2}$ Department of Internal Medicine, Aizawa Hospital; ${ }^{3}$ Department of Internal Medicine, Hata Central Hospital, Matsumoto, Japan

Correspondence and reprints: Dr H Nakama, Department of Public Health, Shinshu University School of Medicine, Asahi 3-1-1,

Matsumoto, 390 Japan. Telephone +81-263-37-2621, fax+81-263-34-4269, e-mail hnakama@sch.md.shinshu-u.ac.jp

Received for publication March 31, 1999. Accepted January 8, 2001 
dépistage immunochimique de sang occulte dans les selles sur un échantillon recueilli lors du toucher rectal. Les deux groupes ont été analysés sur le plan des facteurs individuels, tels la localisation du cancer, la stadification de Dukes et le type histologique des tumeurs.

RÉSULTATS : La prévalence des cancers rectaux a été plus élevée dans le groupe "positif" que dans le groupe "négatif" $(\mathrm{P}<0,05)$, mais on n’a noté aucune différence entre les deux groupes pour ce qui est des autres facteurs.

CONCLUSIONS : Ces observations indiquent que les échantillons recueillis lors d'un toucher rectal ne permettent pas le dépistage du sang occulte dans les selles, surtout s'il est question de néoplasies du côlon proximal.
W hen testing stool for fecal occult blood to detect colorectal cancer, stool is collected by two methods: by testing stool specimens obtained as part of routine screening, and by testing stool specimens obtained during the digital rectal examination as part of the physical examination or for screening purposes. However, the appropriate evaluation of a positive result of a fecal occult blood test on stool obtained during the digital rectal examination is controversial. In addition, little information is available regarding the clinical features of colorectal cancer that are detected in stool specimens obtained during the digital rectal examination.

In the present investigation, to clarify the features of colorectal cancer that produce positive results on an immunochemical fecal occult blood test of stool obtained during the digital rectal examination and to evaluate the significance of testing fecal occult blood in stool obtained during the digital rectal examination, the authors compared the clinicopathological characteristics of these features with the features of colorectal cancer that produce negative results.

\section{MATERIALS AND METHODS}

Subjects for the cross-sectional study were selected from participants in an annual employee medical checkup from April 1, 1990 to March 31, 1999. The two hospitals for the medical checkups were located in central Nagano Prefecture. Physicians from the department of endoscopy of each hospital carried out both colonoscopy and immunochemical fecal occult blood testing using two separate stool samples on all participants, without dietary or medicinal restriction. Participants who had a history of recent rectal bleeding or any symptoms of colorectal diseases before the examination were excluded from the study. Fecal occult blood testing was performed on stool specimens obtained during the digital rectal examination. If the rectal ampulla of the participants was empty, mucus obtained by digital rectal examination was used for the occult blood test. All patients with colorectal cancer diagnosed by colonoscopy were divided into two groups, according to the results of the fecal occult blood test; intergroup comparisons were made in terms of individual factors, such as site, size, Dukes classification and histological type of the cancer lesions (1). The final diagnosis of colorectal cancer was made after surgical or endoscopic removal of tumours.

The immunochemical fecal occult blood test was performed by a trained technician at the hospital laboratory. The principles and procedures of the immunochemical slide Imdia-HemSp (FujiRebio, Japan) (a reversed passive hemagglutination test) (2), which was used in the present investigation, are outlined as follows. The test subjects were first instructed to make a thin fecal smear on the test filter paper. Disks of the specimens from the feces-smeared slides were placed in round wells in a microtitre plate. A diluent was added to extract the specimens from the disks. A portion of the extract was removed and diluted serially in the next three wells of the plate. Erythrocytes coated with antihuman hemoglobin antibody were added to the last wells, and the mixture was incubated at room temperature for $30 \mathrm{~min}$. Samples showing agglutination at a dilution of 1:8 were interpreted as a positive result. The absence of agglutination was interpreted as a negative result. The procedures of this test are uncomplicated, and the cost per slide for each test is approximately US $\$ 4.00$ ( $¥ 440$; average exchange rate was US $\$ 1=¥ 110$ during the period of the investigation).

Statistical analysis was performed by $\chi^{2}$ test, and a twotailed $\mathrm{P}$ value of less than 0.05 was defined as statistically significant.

\section{RESULTS}

Of 9952 subjects who participated in the medical checkup for colorectal cancer, 5830 were male and 4122 were female. The average age of the subjects was 53.6 years (male 54.8 years, female 51.4 years). The results of an immunochemical fecal occult blood test were positive in 786 subjects $(7.9 \%$; $95 \%$ CI $7.4 \%$ to $8.4 \%$; male $7.9 \%$, female $7.8 \%)$. Colorectal cancer was detected by colonoscopy in 64 subjects $(0.6 \%$; $95 \%$ CI $0.4 \%$ to $0.8 \%$; male $0.6 \%$, female $0.6 \%)-39$ subjects $(60.9 \%)$ from the group who tested positive for occult blood and 25 subjects (30.1\%) from the group who tested negative for occult blood, respectively. The sensitivity, specificity, and positive and negative predictive values of the immunochemical fecal occult blood test on stool sampled by digital rectal examination were $61 \%, 92 \%, 5.0 \%$ and $99.8 \%$, respectively (95\% CI $49 \%$ to $73 \%, 91 \%$ to $93 \%, 3.5 \%$ to $6.5 \%$ and $99.7 \%$ to $99.9 \%$, respectively). In the group with positive results on immunochemical fecal occult blood testing, the odds ratio for risk of colorectal cancer was 19.1 (95\% CI 11.5 to 31.7). A total of 319 colonic adenomas were detected during the screening colonoscopy, including 70 large adenomas $(1 \mathrm{~cm}$ or larger) and 249 small adenomas (smaller than $1 \mathrm{~cm}$ ). Of these, 41 large and 160 small adenomas had a positive result, and 29 large and 89 small adenomas had a negative result on immunochemical fecal occult blood testing.

Tumour site: In the group who tested positive for fecal occult blood, the lesion sites were the rectum in 20 patients $(80.0 \%)$, the left side of the colon (sigmoid colon and descending colon) in 15 patients $(48.4 \%)$ and the right side 
TABLE 1

Comparison of tumour site in patients with colorectal cancer who tested positive and those who tested negative for fecal occult blood when stool was obtained during the digital rectal examination

\begin{tabular}{lccr}
\hline & \multicolumn{3}{c}{ Fecal occult blood test result } \\
Tumour site & Positive (\%) & Negative (\%) & \multicolumn{1}{c}{ Total } \\
\hline Rectum & $20(80.0)^{*}$ & $5(20.0)$ & $25(100.0)$ \\
Left side of the colon & $15(48.4)$ & $16(51.6)$ & $31(100.0)$ \\
Right side of the colon & $4(50.0)$ & $4(50.0)$ & $8(100.0)$ \\
Total & $39(60.9)$ & $25(39.1)$ & $64(100.0)$ \\
\hline
\end{tabular}

${ }^{*} P<0.05$ for difference between negative and positive groups

TABLE 2

Comparison of tumour size in patients with colorectal cancer who tested positive and those who tested negative for fecal occult blood when stool was obtained during the digital rectal examination

\begin{tabular}{lccc}
\hline & \multicolumn{3}{c}{ Fecal occult blood test result } \\
Tumour size $\mathbf{( c m )}$ & Positive (\%) & Negative (\%) & Total (\%) \\
\hline$<2$ & $23(60.5)$ & $15(39.5)$ & $38(100.0)$ \\
$\geq 2$ & $16(61.5)$ & $10(38.5)$ & $26(100.0)$ \\
Total & $39(60.9)$ & $25(39.1)$ & $64(100.0)$ \\
\hline
\end{tabular}

of the colon (transverse colon, ascending colon and cecum) in four patients $(50.0 \%)$. In the group who tested negative for fecal occult blood, the lesion sites were the rectum in five patients $(20.0 \%)$, the left side of the colon in 16 patients $(51.6 \%)$ and the right side of the colon in four patients $(50.0 \%)$. Accordingly, the prevalence of rectal cancer was significantly higher in the group who tested positive than in the group who tested negative for fecal occult blood $(\mathrm{P}<0.05)$ (Table 1$)$.

Tumour size: Colorectal cancers were divided into those smaller than $2 \mathrm{~cm}$ and those larger than $2 \mathrm{~cm}$. Among the cancers that were smaller than $2 \mathrm{~cm}$, almost $50 \%$ were neoplastic polyps (early cancer or adenomatous polyps with severe dysplasia). In the group who tested positive for fecal occult blood, 23 lesions (60.5\%) were smaller than $2 \mathrm{~cm}$ and 16 lesions $(61.5 \%)$ were larger than $2 \mathrm{~cm}$. In the group who tested negative for fecal occult blood, 15 lesions (39.5\%) were smaller than $2 \mathrm{~cm}$ and 10 lesions (38.5\%) were larger than $2 \mathrm{~cm}$, suggesting the absence of any significant difference in the distribution of lesion size between the two groups (Table 2).

Dukes stage: Eighteen $(60.0 \%)$ cases of cancer in the positive group and $12(40.0 \%)$ cases in the negative group were classified as Dukes A, suggesting that the frequency of Dukes A cancer was highest in both groups. There was no significant difference in the combined frequency of cases classified as Dukes B and C between the two groups (Table 3).
TABLE 3

Comparison of Dukes stage in patients with colorectal cancer cases who tested positive and those who tested negative for fecal occult blood when stool was obtained during the digital rectal examination

\begin{tabular}{lccc}
\hline & \multicolumn{3}{c}{ Fecal occult blood test result } \\
Dukes stage & Positive (\%) & Negative (\%) & Total(\%) \\
\hline A & $18(60.0)$ & $12(40.0)$ & $30(100.0)$ \\
B & $13(61.9)$ & $8(38.1)$ & $21(100.0)$ \\
C & $8(61.5)$ & $5(38.5)$ & $13(100.0)$ \\
Total & $39(60.9)$ & $25(39.1)$ & $64(100.0)$ \\
\hline
\end{tabular}

TABLE 4

Comparison of histological type in patients with colorectal cancer who tested positive and those who tested negative for fecal occult blood when stool was obtained during the digital rectal examination

\begin{tabular}{lccc}
\hline \multirow{2}{*}{$\begin{array}{l}\text { Histological } \\
\text { type }\end{array}$} & \multicolumn{3}{c}{ Fecal occult blood test result } \\
\hline $\begin{array}{l}\text { Pell differentiated } \\
\text { adenocarcinoma }\end{array}$ & $28(58.3)$ & $20(41.7)$ & $48(100.0)$ \\
$\begin{array}{l}\text { Moderately } \\
\text { differentiated } \\
\text { adenocarcinoma }\end{array}$ & $9(69.2)$ & $4(30.8)$ & $13(100.0)$ \\
$\begin{array}{l}\text { Poorly differentiated } \\
\text { adenocarcinoma }\end{array}$ & $2(66.7)$ & $1(33.3)$ & $3(100.0)$ \\
\begin{tabular}{l} 
Total \\
\hline
\end{tabular} & $39(60.9)$ & $25(39.1)$ & $64(100.0)$ \\
\hline
\end{tabular}

Histological type: In both groups, well differentiated adenocarcinoma accounted for more than half the cases, followed by moderately differentiated and poorly differentiated adenocarcinomas, respectively. There was no significant difference in the distribution of histological type between the two groups (Table 4).

\section{DISCUSSION}

Randomized, controlled trials have shown that fecal occult blood screening for colorectal cancer using patient-collected stool is associated with reduced mortality from colorectal cancer (3-5). Some physicians perform fecal occult blood tests on stool obtained during the digital rectal examination in general practice; however, the clinical impact of this practice is controversial.

Some investigations have demonstrated that patients with positive results on fecal occult blood tests on stool obtained during the digital rectal examination had as many colonic disorders as patients identified through routine screening $(6,7)$. In contrast, a comparative study showed that the stool obtained during the digital rectal examination was unsuitable for the testing of fecal occult blood, because, for colonic neoplasms, the positive predictive 
value of fecal occult blood testing was lower when stool was obtained by this practice than when stool was obtained by patients during routine screening (8). Longstreth (9) editorialized that fecal occult blood testing of stool collected during the digital rectal examination is "a procedure of little value". For this reason, the authors investigated the clinicopathological characteristics of colorectal cancer that produce a positive result on immunochemical fecal occult blood testing of stool obtained during the digital rectal examination, by comparing them with the clinicopathological characteristics of colorectal cancer that produce a negative result.

In general, several factors, including poor sensitivity of the test, inappropriate storage of stool samples, no or intermittent bleeding from cancer, inadequate stool sampling and intraintestinal degeneration of human hemoglobin, may lead to a negative result on fecal occult blood testing in patients with colorectal cancer. For the present study, factors such as lower sensitivity and poor storage of samples can be excluded from the possible causes of negative results because a highly sensitive test kit was used; the cutoff value was adjusted to nearly the upper limit of physiological bleeding (10). Also, an experienced laboratory technician exercised special care during the treatment of specimens when conducting the test.

Accordingly, three factors - intermittent bleeding or small bleeding, intraintestinal degeneration of human hemoglobin and inappropriate sampling - were likely the major reasons for a negative result on immunochemical fecal occult blood testing. Of these factors, intermittent bleeding has been identified as the major possible cause of a negative result of fecal occult blood testing in the study of small colorectal neoplasia $(11,12)$. Also, it has been pointed out that degeneration of hemoglobin is the possible cause of a negative test result in the study of neoplastic lesions in the right side of the colon $(13,14)$. Theoretically, this view is based on the concept that, in neoplasia of the right side of the colon, even if bleeding from lesions exists, hemoglobin peroxidase activity and antigen determinant radicals are destroyed by intestinal flora and enteric juice. As for inappropriate sampling, it has been suggested that, in rectal neoplasia in particular, blood can only adhere to part of the stool. Therefore, it has been emphasized that a negative test result may be obtained, depending on the site of stool sampling (15). To identify rectal neoplasia, it is recommended that stool be sampled in an area longer than $5 \mathrm{~cm}$ and be taken from three regions by brushing (16).

In the present comparative study, the prevalence of rectal cancer was higher in the group who tested positive for fecal occult blood than in the group who tested negative for fecal occult blood; no significant difference was observed in the frequencies of cancer in the right side of the colon between the two groups. These results suggest that appro- priate stool sampling by digital rectal examination may lead to a true positive result on the immunochemical fecal occult blood test in patients with rectal cancer.

\section{CONCLUSIONS}

Stool collected during the digital rectal examination is unsuitable for fecal occult blood testing, especially for the detection of proximal colon neoplasms; special attention should be paid to the method of stool collection to improve the accuracy of fecal occult blood testing.

ACKNOWLEDGEMENTS: The present study was supported in part by Grants-in-Aid for Scientific Research (No 09670384) from the Ministry of Education, Science and Culture of Japan and Cancer Research (No 8-8) from the Ministry of Health and Welfare of Japan.

\section{REFERENCES}

1. Turnbull RB Jr. Cancer of the colon: the five- and ten-year survival rates following resection utilizing the isolation technique. Ann R Coll Surg Engl 1970;46:243-50.

2. Nakama H, Kamijo N, Miyata K, Fattah ASMA, Zhang B, Uehara Y. Sensitivity and specificy of several immunochemical tests for colorectal cancer. Hepatogastroenterology 1998;45:1579-82.

3. Mandel JS, Bond JH, Church TR, et al. Reducing mortality from colorectal cancer by screening for fecal occult blood. N Engl J Med 1993;328:1365-71.

4. Kronborg O, Fenger C, Olsen J, Jorgensen OD, Sondergaard O. Randomised study of screening for colorectal cancer with faecal-occult-blood test. Lancet 1996;348:1467-71.

5. Hardcastle JD, Chamberlain J, Robinson MHE, et al. Randomised controlled trial of faecal-occult-blood screening for colorectal cancer. Lancet 1996;348:1472-7.

6. Eisner MS, Lewis JH. Diagnostic yield of a positive fecal occult blood test found on digital rectal examination: does the finger count? Arch Intern Med 1991;151:2180-4.

7. Brint SL, DiPalna JA, Herrera JL. Is a Hemoccult positive rectal examination clinically significant? South Med J 1993;86:601-3.

8. Marks RD, Hall G, Vcerapalli R, Singh S. Fecal occult blood testing: does the stool collection method affect outcome? Gastroenterology 1997;112(Suppl):A29. (Abst)

9. Longstreth FG. Checking for the 'occult' with a finger: a procedure of little value. J Clin Gastroenterol 1988;10:133-4.

10. Yoshida Y, Saito H, Tsuchida S, et al. A simple sensitive immunologic fecal occult blood test suitable for mass screening for colorectal cancer. Gastroenterology 1986;90:1699. (Abst)

11. Simon JB. Occult blood screening for colorectal cancer: a critical review. Gastroenterology 1985;88:820-37.

12. Ahlquist DA. Occult gastrointestinal bleeding. In: Yamada T, ed. Textbook of Gastroenterology. Philadelphia: JB Lippincott, 1995:699-717.

13. Goldschmiedt M, Ahlquist DA, Wieand HS, et al. Measurement of degraded fecal hemoglobin-heme to estimate gastrointestinal site of occult bleeding: appraisal of its clinical utility. Dig Dis Sci 1988;33:605-8.

14. Mandel JS, Bond JH, Bradley M, et al. Sensitivity, specificity, and positive predictivity of the Hemoccult test in screening for colorectal cancer: the University of Minnesota's colon cancer. Gastroenterology 1989;97:597-600.

15. Herzog P, Holtermuller KH, Preiss J, et al. Fecal blood loss in patients with colonic polyps: a comparison of measurements with 51chromium-labeled erythrocytes and with the Hemoccult test. Gastroenterology 1982;29:2061-7.

16. Imai $S$. The distribution of the positive spots for immunological occult blood test in the feces of colorectal cancer. J Jpn Soc Coloproctol 1990;43:1142-53. 


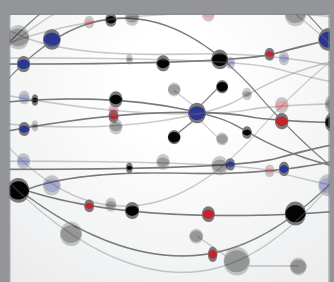

The Scientific World Journal
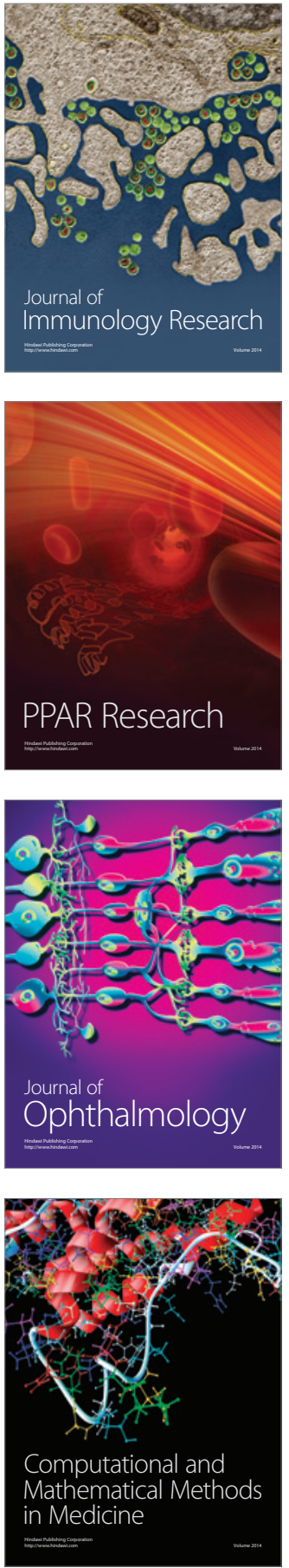

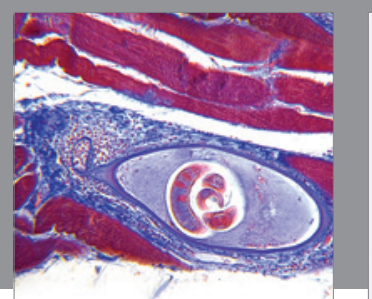

Gastroenterology Research and Practice

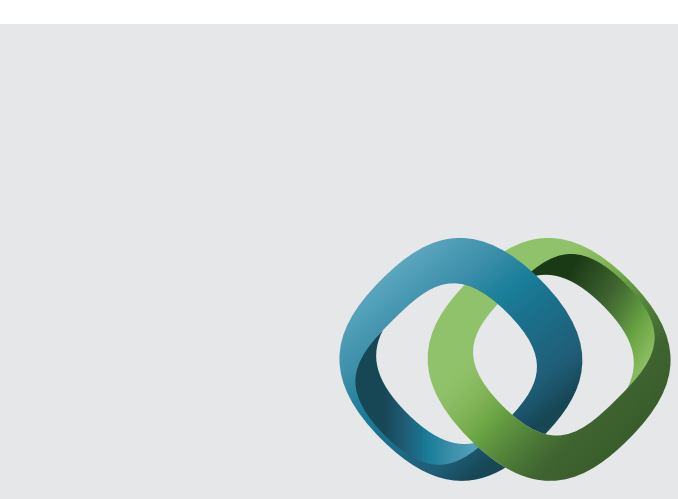

\section{Hindawi}

Submit your manuscripts at

http://www.hindawi.com
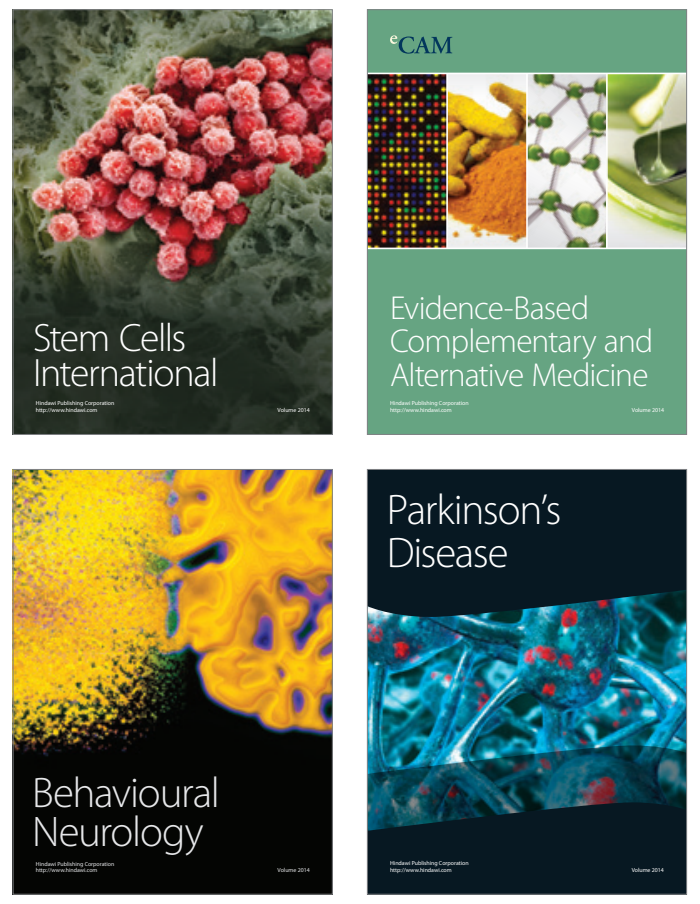
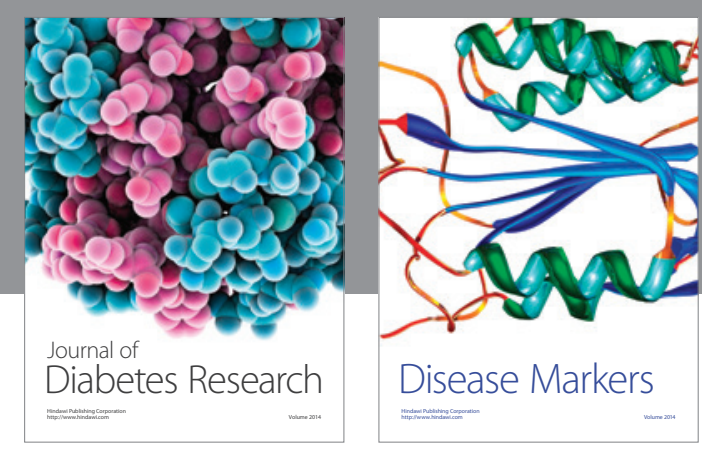

Disease Markers
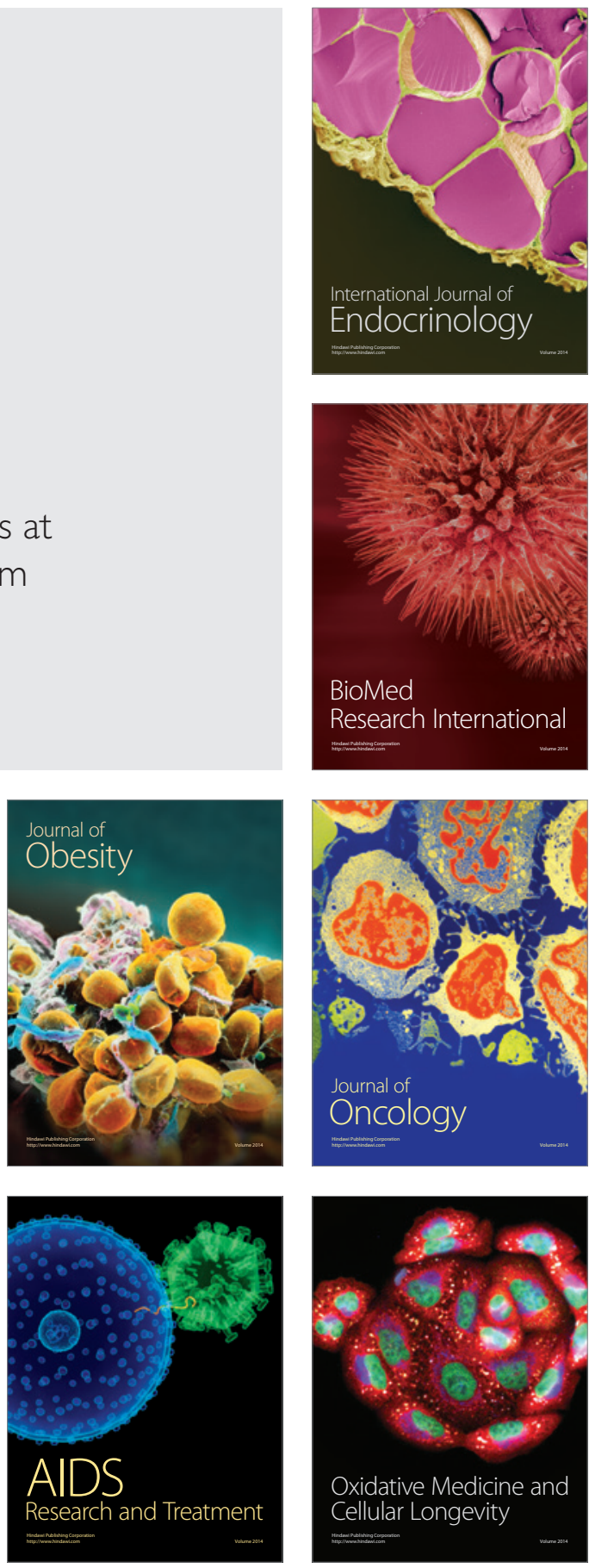